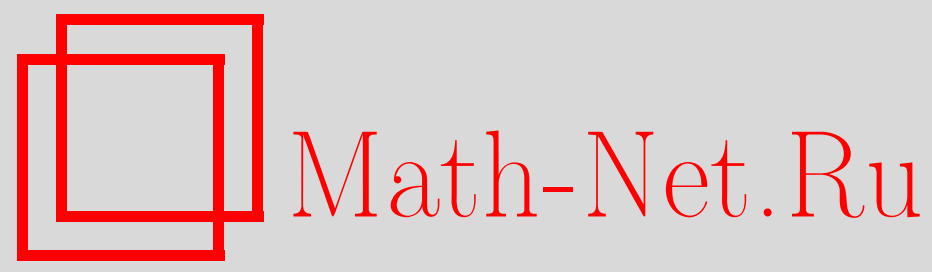

Л. В. Крицков, Оценка образов Фурье по системе обобщенных собственных функций оператора Шредингера с потенциалом штуммелевского типа, Матем. заметки, 1999, том 65, выпуск 4, 542-551

DOI: https://doi.org/10.4213/mzm1080

Использование Общероссийского математического портала Math-Net.Ru подразумевает, что вы прочитали и согласны с пользовательским соглашением http://www . mathnet.ru/rus/agreement

Параметры загрузки:

IP : 54.80 .97 .219

26 апреля 2023 г., $17: 16: 30$

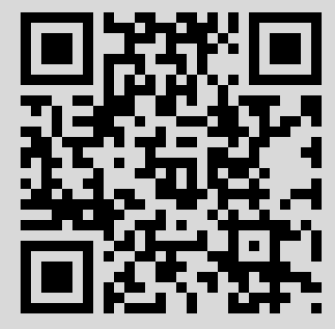




\section{ОЦЕНКА ОБРАЗОВ ФУРЬЕ ПО СИСТЕМЕ ОБОБЩЕННЫХ СОБСТВЕННЫХ ФУНКЦИЙ ОПЕРАТОРА ШРЁДИНГЕРА С ПОТЕНЦИАЛОМ ШТУММЕЛЕВСКОГО ТИПА}

\section{Л.В. Крицков}

Пусть $\mathscr{A}$ - неотрицательное самосопряженное расширение в $\mathbb{R}^{N}(N \geqslant 1)$ формального дифференциального оператора $-\Delta u+q(x) u$ с потенциалом $q(x)$, удовлетворяющим при $N=1,2,3$ условию

$$
\sup _{x \in \mathbb{R}^{N}} \int_{|x-y| \leqslant 1}|q(y)|^{2} d y<\infty
$$

и при $N \geqslant 4$ условию

$$
\sup _{x \in \mathbb{R}^{N}} \int_{|x-y| \leqslant 1}|x-y|^{4-N} \varkappa(|x-y|)|q(y)|^{2} d y<\infty,
$$

в котором неотрицательная функция $\varkappa(r)$ такова, что $\int_{0}^{1}(\varkappa(r) r)^{-1} d r<\infty$. Для каждого $\alpha \in(0,2]$ установлена оценка для обобщенных образов Фурье произвольной функции $f \in L_{2}^{\alpha}\left(\mathbb{R}^{N}\right)$ вида

$$
\sum_{i=1}^{m} \int_{0}^{\infty}\left|\widehat{f}_{i}(\lambda)\right|^{2}(1+\lambda)^{\alpha} d \rho(\lambda) \leqslant M\|f\|_{L_{2}^{\alpha}\left(\mathbb{R}^{N}\right)}^{2} .
$$

Если дополнительно $\lim _{r \rightarrow 0+0} \varkappa(r)=+\infty$, то наряду с этой обоснована аналогичная оценка снизу.

Библиография: 11 названий.

Изучается самосопряженный оператор Шрёдингера $\mathscr{A}$, определяемьй во всем пространстве $\mathbb{R}^{N}(N \geqslant 1)$ дифференциальным выражением

$$
A u=-\Delta u+q(x) u
$$

в котором потенциал $q(x)$ удовлетворяет условию штуммелевского типа $[1$, c. 13$]$ :

$$
\sup _{x \in \mathbb{R}^{N}} \int_{|x-y| \leqslant 1}|q(y)|^{2} d y<\infty,
$$

Работа выполнена в рамках программы ESPRIT сотрудничества между EC и Россией, грант № 21042 CTIAC, и при частичной поддержке Российского фонда фундаментальных исследований, грант № 96-15-96097. 
если $N=1,2,3$, и

$$
\sup _{x \in \mathbb{R}^{N}} \int_{|x-y| \leqslant 1}|x-y|^{4-N} \varkappa(|x-y|)|q(y)|^{2} d y<\infty
$$

если $N \geqslant 4$. Здесь $\varkappa(r)$ - произвольная неотрищательная функция, удовлетворяющая условию

$$
\int_{0}^{1}(\varkappa(r) r)^{-1} d r<\infty
$$

Если потенциал в (1) удовлетворяет условию (2), (3), то, как следует из [1, с. 15], самосопряженньй оператор $\mathscr{A}$ может быть определен замыканием минимального оператора, порождаемого выражением (1) на множестве $C_{0}^{\infty}\left(\mathbb{R}^{N}\right)$, и является ограниченным снизу. В силу инвариантности классов (2) и (3) относительно прибавления произвольной константы можно считать, что оператор $\mathscr{A}$ неотрицателен. Из [2], [3] следует, что пространство $L_{2}\left(\mathbb{R}^{N}\right)$ допускает упорядоченное спектральное представление относительно оператора $\mathscr{A}$, характеризуемое мерой $\rho(\lambda)$, кратностью $m \leqslant \infty$ и обобщенными собственными функциями $u_{i}(x, \lambda), i=1, \ldots, m$, так что для каждой функции

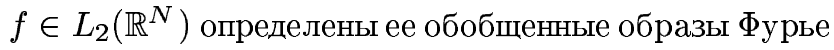

$$
\widehat{f}_{i}(\lambda)=\int_{\mathbb{R}^{N}} f(x) u_{i}(x, \lambda) d x, \quad i=1, \ldots, m
$$

В данной работе доказана следующая

Теорема. Пусть $\mathscr{A}$ - неотрицательный самосопряженный оператор Шрёдингера (1) с потенииалом, удовлетворяющим в $\mathbb{R}^{N}$ условию (2), (3). Тогда можно указать постоянную $M>0$ такую, что для каждой функиии $f$ из класса Соболева-Лиувилля $L_{2}^{\alpha}\left(\mathbb{R}^{N}\right)$ с произвольным порядком дифферениируемости $\alpha \in(0,2]$ выполнена оченка

$$
\sum_{i=1}^{m} \int_{0}^{\infty}\left|\widehat{f}_{i}(\lambda)\right|^{2}(1+\lambda)^{\alpha} d \rho(\lambda) \leqslant M\|f\|_{L_{2}^{\alpha}\left(\mathbb{R}^{N}\right)}^{2}
$$

Если функиия $\varkappa(r)$ в (3) удовлетворяет дополнительному условию $\varkappa(r) \rightarrow+\infty$ nри $r \rightarrow 0+0$, то наряду с оченкой сверху (5) выполнена аналогичная оценка снизу c некоторой постоянной $m>0$.

Оценки образов Фурье позволяют исследовать абсолютную сходимость спектральных разложений, отвечающих оператору $\mathscr{A}$, в равномерной во всем пространстве $\mathbb{R}^{N}$ метрике. Отметим, что одномерный случай $(N=1)$ рассматривался в [4]-[7].

1. Доказательство оценки сверху. Положим $\nu=(N-2) / 2$ и обозначим через $\tau_{\alpha / 2}(r)$ ядро Бесселя-Макдональда:

$$
\tau_{\alpha / 2}(r)=2^{1-\alpha / 2}\left(\left(\frac{2}{\pi}\right)^{\nu+1} \Gamma\left(\frac{\alpha}{2}\right)\right)^{-1} r^{-(\nu+1)+\alpha / 2} K_{(\nu+1)-\alpha / 2}(r)
$$


Тогда если $f \in L_{2}^{\alpha}\left(\mathbb{R}^{N}\right)$, то существует $h \in L_{2}\left(\mathbb{R}^{N}\right)$ такая, что

$$
f(x)=\int_{\mathbb{R}^{N}} \tau_{\alpha / 2}(|x-y|) h(y) d y
$$

Из свойств модифицированных функций Бесселя [8, гл. 7] вытекает, что ядро (6) удовлетворяет следующим оценкам:

$$
c_{1} r^{-(2 \nu+3-\alpha) / 2} e^{-r} \leqslant \tau_{\alpha / 2}(r) \leqslant C_{1} r^{-(2 \nu+3-\alpha) / 2} e^{-r}
$$

при $N \geqslant 1,0<\alpha \leqslant 2, r \geqslant 1$;

$$
c_{2} r^{-(2 \nu+2-\alpha)} \leqslant \tau_{\alpha / 2}(r) \leqslant C_{2} r^{-(2 \nu+2-\alpha)}
$$

при $N \geqslant 1,0<\alpha \leqslant 2, N-\alpha>0,0<r \leqslant 1$;

$$
c_{3} \ln \frac{e}{r} \leqslant \tau_{\alpha / 2}(r) \leqslant C_{3} \ln \frac{e}{r}
$$

при $N=\alpha=2$ или $N=\alpha=1$ и $0<r \leqslant 1$;

$$
c_{4} \leqslant \tau_{\alpha / 2}(r) \leqslant C_{4}
$$

при $N=1,1<\alpha \leqslant 2,0<r \leqslant 1$.

Положим

$$
\begin{gathered}
w_{\nu}\left(z_{1}, z_{2}\right)=J_{\nu}\left(z_{1}\right) Y_{\nu}\left(z_{2}\right)-J_{\nu}\left(z_{2}\right) Y_{\nu}\left(z_{1}\right) \\
W_{\nu}(\rho, \mu)=\frac{\pi \rho^{-\nu}}{2} \int_{\rho}^{\infty} r^{\nu+1} \tau_{\alpha / 2}(r) w_{\nu}(\mu \rho, \mu r) d r, \quad \mu=\sqrt{\lambda}
\end{gathered}
$$

Для образов Фурье функции $f \in L_{2}^{\alpha}\left(\mathbb{R}^{N}\right)$ по системе обобщенных собственных функций оператора $\mathscr{A}$ имеет место представление (ср. с [7, формула (12)])

$$
\widehat{f}_{i}(\lambda)=(1+\lambda)^{-\alpha / 2} \widehat{h}_{i}(\lambda)-\int_{\mathbb{R}^{N}} W_{\nu}(|z|, \mu) \int_{\mathbb{R}^{N}} h(y-z) q(y) u_{i}(y, \lambda) d y d z .
$$

Обозначим второе слагаемое в правой части этого равенства через $\left(T_{i} q\right)(\lambda)$. Тогда из (14) вытекает, что левая часть (5) не превосходит

$$
2 \sum_{i=1}^{m} \int_{0}^{\infty}\left|\widehat{h}_{i}(\lambda)\right|^{2} d \rho(\lambda)+2 \sum_{i=1}^{m} \int_{0}^{\infty}\left|\left(T_{i} q\right)(\lambda)\right|^{2}(1+\lambda)^{\alpha} d \rho(\lambda) \equiv S_{1}+S_{2}
$$

Первое слагаемое $S_{1}$ в $(15)$ в силу обобщенного равенства Парсеваля (упорядоченное спектральное представление пространства $L_{2}\left(\mathbb{R}^{N}\right)$ относительно $\mathscr{A}$ изометрично) есть $2\|h\|_{L_{2}\left(\mathbb{R}^{N}\right)}^{2}=2\|f\|_{L_{2}^{\alpha}\left(\mathbb{R}^{N}\right)}^{2}$, а для оценки второго применим следующую лемму, которую докажем в п. 3 . 
Лемма. Для всех $\nu \geqslant-1 / 2, \rho>0, \mu \geqslant 0 u 0<\alpha \leqslant 2$ выполнена оиенка

$$
\left|W_{\nu}(\rho, \mu)\right| \leqslant C_{5}\left(1+\mu^{2}\right)^{-\alpha / 2} \tau_{1}(\rho) .
$$

Из оценки (16) следует, что

$$
S_{2} \leqslant C_{5}^{2} \sum_{i=1}^{m} \int_{0}^{\infty}\left(\int_{\mathbb{R}^{N}} \tau_{1}(|z|)\left|\int_{\mathbb{R}^{N}} h(y-z) q(y) u_{i}(y, \lambda) d y\right| d z\right)^{2} d \rho(\lambda) .
$$

Разобьем область интегрирования по переменной $z$ в правой части (17) на две: $|z| \leqslant 1$ и $|z| \geqslant 1,-$ и обозначим соответствующие слагаемые через $I_{1}$ и $I_{2}$. Для $I_{2}$ применим неравенство Коши-Буняковского, обобщенное равенство Парсеваля и учтем (8):

$$
I_{2} \leqslant O(1) \int_{|z| \geqslant 1} \tau_{1}(|z|) \int_{\mathbb{R}^{N}}|h(y-z)|^{2}|q(y)|^{2} d y d z .
$$

Производя замену переменной и меняя порядок интегрирования, придем к оценке

$$
I_{2} \leqslant O(1)\|h\|_{L_{2}\left(\mathbb{R}^{N}\right)}^{2} \sup _{x \in \mathbb{R}^{N}} \int_{|x-y| \geqslant 1} \tau_{1}(|x-y|)|q(y)|^{2} d y .
$$

Так как в силу (8) $\tau_{1}(r)=O(1) e^{-r}$ при $r \geqslant 1$, то

$$
\sup _{x \in \mathbb{R}^{N}} \int_{|x-y| \geqslant 1} \tau_{1}(|x-y|)|q(y)|^{2} d y \leqslant O(1) \sum_{l=1}^{\infty} e^{-l} \sup _{x \in \mathbb{R}^{N}} \int_{l \leqslant|x-y|<l+1}|q(y)|^{2} d y .
$$

Шаровой слой $\{l \leqslant|x-y| \leqslant l+1\}$ можно покрыть не более, чем const $\cdot l^{N-1}$ единичными шарами, поэтому правая часть последней оценки не превосходит

$$
O(1) \sup _{x \in \mathbb{R}^{N}} \int_{|x-y| \geqslant 1}|q(y)|^{2} d y
$$

а из (2) и (3) следует, что эта величина конечна при любом $N \geqslant 1$. Тем самым, из (18) следует, что

$$
I_{2} \leqslant O(1)\|h\|_{L_{2}\left(\mathbb{R}^{N}\right)}^{2}
$$

Из оценок (9)-(11) следует, что при $N=1,2,3 \tau_{1}(|z|) \in L_{2}(|z| \leqslant 1)$. Поэтому, применяя к $I_{1}$ сначала неравенство Коши-Буняковского, а затем обобщенное равенство Парсеваля, получим

$I_{1} \leqslant O(1) \int_{|z| \leqslant 1} \int_{\mathbb{R}^{N}}|h(y-z)|^{2}|q(y)|^{2} d y d z \leqslant O(1)\|h\|_{L_{2}\left(\mathbb{R}^{N}\right)}^{2} \sup _{x \in \mathbb{R}^{N}} \int_{|x-y| \geqslant 1}|q(y)|^{2} d y$.

Кроме того, из (9) получим, что при $N \geqslant 4$ и $r \leqslant 1$

$$
\tau_{1}(r)=O(1) r^{2-N} \equiv O(1) r^{-N / 2}(\varkappa(r))^{-1 / 2} r^{2-N / 2}(\varkappa(r))^{1 / 2} .
$$

Тогда аналогично (20) имеем

$$
\begin{aligned}
I_{1} & \leqslant O(1) \int_{|z| \leqslant 1}\left(|z|^{N} \varkappa(|z|)\right)^{-1} d z \cdot \int_{|z| \leqslant 1}|z|^{4-N} \varkappa(|z|) \int_{\mathbb{R}^{N}}|h(y-z)|^{2}|q(y)|^{2} d y d z \\
& \leqslant O(1)\|h\|_{L_{2}\left(\mathbb{R}^{N}\right)}^{2} \int_{0}^{1}(\varkappa(r) r)^{-1} d r \cdot \sup _{x \in \mathbb{R}^{N}} \int_{|x-y| \leqslant 1}|x-y|^{4-N} \varkappa(|x-y|)|q(y)|^{2} d y .
\end{aligned}
$$

Из оценок (19)-(21) и условий (2)-(4) следует, что $S_{2}=O(1)\|f\|_{L_{2}^{\alpha}\left(\mathbb{R}^{N}\right)}^{2}$. Оценка (5) полностью доказана. 
2. Доказательство оценки снизу. Вьполним преобразование независимой переменной в выражении (1): $x=\varepsilon y$, где $\varepsilon \in(0,1)$ пока произвольно. Рассмотрим дифференциальное выражение

$$
A_{\varepsilon} v=-\Delta v+q_{\varepsilon}(y) v, \quad y \in \mathbb{R}^{N}
$$

в котором потенциал $q_{\varepsilon}(y)$ задается равенством $q_{\varepsilon}(y)=\varepsilon^{2} q(\varepsilon y)$, и порождаемый этим выражением самосопряженньй оператор $\mathscr{A}_{\varepsilon}$. Упорядоченные спектральные представления пространства $L_{2}\left(\mathbb{R}^{N}\right)$ относительно операторов $\mathscr{A}_{\text {и }} \mathscr{A}_{\varepsilon}$ связаны, очевидно, следующим образом. Положим $\lambda_{\varepsilon}=\varepsilon^{2} \lambda$. Выберем обобщенными собственными функциями оператора $\mathscr{A}_{\varepsilon}$ функции $v_{i}\left(y, \lambda_{\varepsilon}\right)=u_{i}\left(\varepsilon y, \varepsilon^{-2} \lambda_{\varepsilon}\right)$. Если обобщенньй образ Фурье функции $F(y)$ относительно системы этих функций обозначить через $\widetilde{F}_{i}\left(\lambda_{\varepsilon}\right)$, то обобщенный образ Фурье функции $f(x)=F(x / \varepsilon)$ будет удовлетворять равенству $\widehat{f}_{i}(\lambda)=\varepsilon^{N} \widetilde{F}_{i}\left(\varepsilon^{2} \lambda\right)$. Из равенств Парсеваля следует, что для спектральных мер этих упорядоченных представлений имеет место соотношение $\rho_{\varepsilon}\left(\varepsilon^{2} \lambda\right)=\varepsilon^{N} \rho(\lambda)$.

Рассмотрим произвольную функцию $f \in L_{2}^{\alpha}\left(\mathbb{R}^{N}\right)$ и функцию $h \in L_{2}\left(\mathbb{R}^{N}\right)$, связанную c $f$ равенством (7). Изучим свойства обобщенных образов Фурье функции $F(y)=f(\varepsilon y)$. Пусть $h^{(\varepsilon)}(y) \in L_{2}\left(\mathbb{R}^{N}\right)$ такова, что

$$
F(y)=\int_{\mathbb{R}^{N}} \tau_{\alpha / 2}(|y-z|) h^{(\varepsilon)}(z) d z
$$

Тогда аналогично (14) получим

$$
\widetilde{F}_{i}\left(\lambda_{\varepsilon}\right)=\left(1+\lambda_{\varepsilon}\right)^{-\alpha / 2} \widetilde{h}_{i}^{(\varepsilon)}\left(\lambda_{\varepsilon}\right)+\left(T_{i}^{(\varepsilon)} q_{\varepsilon}\right)\left(\lambda_{\varepsilon}\right)
$$

здесь $T_{i}^{(\varepsilon)}$ имеет тот же смысл, что и в $(14)$, но с заменой $h$ на $h^{(\varepsilon)}$. На основании $(22)$ и равенства Парсеваля придем к следующему неравенству:

$$
\begin{aligned}
& \sum_{i=1}^{m} \int_{0}^{\infty}\left|\widetilde{F}_{i}\left(\lambda_{\varepsilon}\right)\right|^{2}\left(1+\lambda_{\varepsilon}\right)^{\alpha} d \rho_{\varepsilon}\left(\lambda_{\varepsilon}\right) \\
& \quad \geqslant \frac{1}{2} \sum_{i=1}^{m} \int_{0}^{\infty}\left|\widetilde{h}_{i}^{(\varepsilon)}\left(\lambda_{\varepsilon}\right)\right|^{2} d \rho_{\varepsilon}\left(\lambda_{\varepsilon}\right)-\sum_{i=1}^{m} \int_{0}^{\infty}\left|\left(T_{i}^{(\varepsilon)} q_{\varepsilon}\right)\left(\lambda_{\varepsilon}\right)\right|^{2}\left(1+\lambda_{\varepsilon}\right)^{\alpha} d \rho_{\varepsilon}\left(\lambda_{\varepsilon}\right) \\
& \quad \equiv \frac{1}{2}\left\|h^{(\varepsilon)}\right\|_{L_{2}\left(\mathbb{R}^{N}\right)}^{2}-I_{3} .
\end{aligned}
$$

Повторим для $I_{3}$ все рассуждения, проведенные в п. 1. Получим аналогично (19)-(21) следующую оценку:

$$
\begin{aligned}
I_{3} \leqslant & O(1)\left\|h^{(\varepsilon)}\right\|_{L_{2}\left(\mathbb{R}^{N}\right)}^{2}\left\{\sup _{x \in \mathbb{R}^{N}} \int_{|x-y| \leqslant 1}\left|q_{\varepsilon}(y)\right|^{2} d y\right. \\
& \left.+\int_{0}^{1}(\varkappa(\varepsilon r) r)^{-1} d r \cdot \sup _{x \in \mathbb{R}^{N}} \int_{|x-y| \leqslant 1}|x-y|^{4-N} \varkappa(\varepsilon|x-y|)\left|q_{\varepsilon}(y)\right|^{2} d y\right\},
\end{aligned}
$$


если $N \geqslant 4$, и ту же оценку, однако без второго слагаемого в фигурных скобках, если $N=1,2,3$.

При $N=1,2,3$ имеем (здесь и далее $x_{1}=\varepsilon x$ )

$$
\int_{|x-y| \leqslant 1}\left|q_{\varepsilon}(y)\right|^{2} d y \leqslant \varepsilon^{4-N} \int_{\left|x_{1}-y_{1}\right| \leqslant 1}\left|q\left(y_{1}\right)\right|^{2} d y_{1}
$$

При $N \geqslant 4$ получим, во-первых,

$$
\begin{aligned}
& \int_{|x-y| \leqslant 1}\left|q_{\varepsilon}(y)\right|^{2} d y=\varepsilon^{4-N} \int_{\left|x_{1}-y_{1}\right| \leqslant \varepsilon}\left|q\left(y_{1}\right)\right|^{2} d y_{1} \\
& \leqslant \sup _{0<r \leqslant \varepsilon}(\varkappa(r))^{-1} \cdot \int_{\left|x_{1}-y_{1}\right| \leqslant 1}\left|x_{1}-y_{1}\right|^{4-N} \varkappa\left(\left|x_{1}-y_{1}\right|\right)\left|q\left(y_{1}\right)\right|^{2} d y_{1}
\end{aligned}
$$

и, во-вторых,

$$
\begin{aligned}
\int_{|x-y| \leqslant 1}|x-y|^{4-N} \varkappa(\varepsilon|x-y|)\left|q_{\varepsilon}(y)\right|^{2} d y \\
\leqslant \int_{\left|x_{1}-y_{1}\right| \leqslant 1}\left|x_{1}-y_{1}\right|^{4-N} \varkappa\left(\left|x_{1}-y_{1}\right|\right)\left|q\left(y_{1}\right)\right|^{2} d y_{1} .
\end{aligned}
$$

Так как

$$
\sup _{0<r \leqslant \varepsilon}(\varkappa(r))^{-1} \rightarrow 0 \quad \text { и } \quad \int_{0}^{1}(\varkappa(\varepsilon r) r)^{-1} d r \rightarrow 0
$$

при $\varepsilon \rightarrow 0+0$, в силу соотношений (25)-(27) правая часть оценки (24) есть $o(1) \times$ $\left\|h^{(\varepsilon)}\right\|_{L_{2}\left(\mathbb{R}^{N}\right)}^{2}$ при $\varepsilon \rightarrow 0+0$. Выбирая достаточно малой величину $\varepsilon \in(0,1)$, на основании неравенства (23) получим

$$
\sum_{i=1}^{m} \int_{0}^{\infty}\left|\widetilde{F}_{i}\left(\lambda_{\varepsilon}\right)\right|^{2}\left(1+\lambda_{\varepsilon}\right)^{\alpha} d \rho_{\varepsilon}\left(\lambda_{\varepsilon}\right) \geqslant \frac{1}{4}\left\|h^{(\varepsilon)}\right\|_{L_{2}\left(\mathbb{R}^{N}\right)}^{2}
$$

Из этой оценки стандартными рассуждениями [7] вьводится требуемая оценка снизу.

3. Доказательство леммы. Прежде всего отметим, что при $\alpha=2$ с учетом (6) и известньх интегральных формул для цилиндрических функций $[8$, c. 104] получим $W_{\nu}(\rho, \mu)=\left(1+\mu^{2}\right)^{-1} \tau_{1}(\rho)$. Тем самьг, достаточно рассмотреть только $\alpha \in(0,2)$.

Пусть сначала $0<\mu \leqslant 1$. В этом случае вместо (16) достаточно доказать оценку

$$
\left|W_{\nu}(\rho, \mu)\right| \leqslant C_{5} \tau_{1}(\rho) .
$$

Проинтегрируем правую часть (13) по частям:

$$
W_{\nu}(\rho, \mu)=\frac{\pi}{2} \mu \rho^{-\nu} \int_{\rho}^{\infty} r^{\nu}\left(J_{\nu}(\mu \rho) Y_{\nu-1}(\mu r)-Y_{\nu}(\mu \rho) J_{\nu-1}(\mu r)\right) \int_{r}^{\infty} \xi \tau_{\alpha / 2}(\xi) d \xi d r
$$


и заметим, что [8, с. 103]

$$
\int_{r}^{\infty} \xi \tau_{\alpha / 2}(\xi) d \xi=\alpha \tau_{(\alpha+2) / 2}(r)
$$

Если $\rho \geqslant 1 / \mu$, то требуемая оценка (28) следует из (9) и оценок цилиндрических функций при больших значениях аргумента. Если же $0<\rho \leqslant 1 / \mu$, то необходимо учесть, что $\tau_{(\alpha+2) / 2}(r)$ (в силу оценок (8)-(11)) есть $O(1) r \tau_{1}(r)$ при $r \geqslant 1$ и любом $\nu \geqslant-1 / 2, O(1)$ при $r \leqslant 1$ и $\nu=-1 / 2$ или $\nu=0, O(1) r^{\alpha-2 \nu}$ при $r \leqslant 1$ и $\nu \geqslant 1$ и является суммируемой на $(0,1)$ при $\nu=1 / 2$. Оценка $(16)$ при $0<\mu \leqslant 1$ полностью доказана.

Пусть теперь $\mu \geqslant 1$. Если $\rho \geqslant 1$, то, интегрируя правую часть (13) иным нежели в (29) способом, получим

$W_{\nu}(\rho, \mu)=\mu^{-2} \tau_{\alpha / 2}(\rho)-\frac{\pi}{2 \mu \rho^{\nu}} \int_{\rho}^{\infty} r^{\nu+1} \tau_{\alpha / 2}^{\prime}(r)\left(J_{\nu}(\mu \rho) Y_{\nu+1}(\mu r)-Y_{\nu}(\mu \rho) J_{\nu+1}(\mu r)\right) d r$

Требуемая оценка (28) вытекает из последнего соотношения, если принять во внимание асимптотические оценки цилиндрических функций при больших значениях аргумента и легко проверяемую (с помощью (6) и формул дифференцирования [8, с. 91]) оценку $\tau_{\alpha / 2}^{\prime}(r)=O(1) r^{-(2 \nu+3-\alpha) / 2} e^{-r}, r \geqslant 1$.

В случае $1 / \mu \leqslant \rho \leqslant 1$ аналогичные рассуждения проходят, если интеграл в правой части (31) взять еще раз по частям:

$$
\begin{aligned}
& W_{\nu}(\rho, \mu)=\mu^{-2} \tau_{\alpha / 2}(\rho)-2(\nu+1) \mu^{-4} \rho^{-1} \tau_{\alpha / 2}^{\prime}(\rho) \\
& \quad+\frac{\pi}{2} \mu^{-2} \rho^{-\nu} \int_{\rho}^{\infty} r^{\nu}\left(r \tau_{\alpha / 2}^{\prime \prime}(r)-\tau_{\alpha / 2}^{\prime}(r)\right)\left(J_{\nu}(\mu \rho) Y_{\nu+2}(\mu r)-Y_{\nu}(\mu \rho) J_{\nu+2}(\mu r)\right) d r
\end{aligned}
$$

и учесть, что $-\tau_{\alpha / 2}^{\prime}(\rho)$ и $\tau_{\alpha / 2}^{\prime \prime}(\rho)$ положительны при $\rho>0$.

Случай $0<\rho \leqslant 1 / \mu$ требует более подробного анализа.

При $\nu=-1 / 2$ подставим в правую часть (13) явные формулы для цилиндрических функций:

$$
W_{-1 / 2}(\rho, \mu)=\mu^{-1} \int_{\rho}^{\infty} \tau_{\alpha / 2}(r) \sin \mu(r-\rho) d r
$$

В силу формул $(1.12 .40)$ и $(2.12 .50)$ из $[9$, с. 52,102$]$ и $(22)$ из $[10$, с. 76$]$ имеем

$$
\begin{aligned}
& \mu^{-1} \int_{0}^{\infty} \tau_{\alpha / 2}(r) \sin \mu(r-\rho) d r=2^{(\alpha-1) / 2} \mu^{-1}\left(1+\mu^{2}\right)^{-\alpha / 2} \\
& \quad \times\left(\cos \mu \rho \cdot \Gamma\left(\frac{\alpha+1}{2}\right)\left(\frac{\mu^{2}}{1+\mu^{2}}\right)^{1 / 2} F\left(\frac{\alpha+1}{2}, \frac{1}{2} ; \frac{3}{2} ; \frac{\mu^{2}}{1+\mu^{2}}\right)-\sin \mu \rho \cdot \frac{\sqrt{\pi}}{2} \Gamma\left(\frac{\alpha}{2}\right)\right),
\end{aligned}
$$

что есть $O(1) \mu^{-\alpha}$, а следовательно, и $O(1) \mu^{-\alpha} \tau_{1}(\rho)$ в силу $(11)$. Требуемая оценка для интеграла

$$
\mu^{-1} \int_{0}^{\rho} \tau_{\alpha / 2}(r) \sin \mu(r-\rho) d r
$$

вытекает непосредственно из (9)-(11). 
При $\nu=0$ вьполним в (13) преобразование $\int_{\rho}^{\infty}=\int_{0}^{\infty}-\int_{0}^{\rho}$ и учтем, что в силу формул $(8.13 .3)$ и $(8.13 .4)$ из $[11$, с. 61$]$

$$
\begin{aligned}
2 \pi \int_{0}^{\infty} r \tau_{\alpha / 2}(r) J_{0}(\mu r) d r= & \left(1+\mu^{2}\right)^{-\alpha / 2} \\
2 \pi \int_{0}^{\infty} r \tau_{\alpha / 2}(r) Y_{0}(\mu r) d r= & \left(\pi \Gamma\left(\frac{\alpha}{2}\right)\right)^{-1}\left(1+\mu^{2}\right)^{-\alpha / 2} \\
& \times\left(\Gamma^{\prime}\left(\frac{\alpha}{2}\right)+\left(\ln \frac{\mu^{2}}{1+\mu^{2}}-\Phi^{\prime}(0)\right) \Gamma\left(\frac{\alpha}{2}\right)\right),
\end{aligned}
$$

где

$$
\Phi(z)=\frac{F\left(\alpha / 2,-z ; 1-z ; \mu^{2} /\left(1+\mu^{2}\right)\right)}{\Gamma(1-z)} .
$$

Так как при $\mu \geqslant 1 \mu^{2} /\left(1+\mu^{2}\right) \in[1 / 2,1)$ и функщия $\Phi(z)$ аналитична по $z[10$, c. 74,80$]$, то, учитьвая $(32),(33)$, асимптотические оценки для $J_{0}(z), Y_{0}(z)$ и ядра $\tau_{\alpha / 2}(r)$ при $\nu=0$, получим $W_{0}(\rho, \mu)=O(1) \mu^{-\alpha} \ln (e / \rho)=O(1) \mu^{-\alpha} \tau_{1}(\rho)$.

Если при $\nu \geqslant 1 / 2$ к правой части (13) применить асимптотические оценки для цилиндрических функций, а также (8) и (9), то требуемая оценка (16) имеет место при всех $\alpha \in(0,2)$, если $\nu \geqslant 3 / 2$, при $\alpha \in(0,3 / 2)$, если $\nu=1$, и при $\alpha \in(0,1)$, если $\nu=1 / 2$.

Пусть $\nu=1$ и $3 / 2 \leqslant \alpha<2$. Воспользуемся формулами $(29)$ и $(30)$ :

$$
W_{1}(\rho, \mu)=\frac{\pi}{2} \alpha \mu \rho^{-1} \int_{\rho}^{\infty} r\left(J_{1}(\mu \rho) Y_{0}(\mu r)-Y_{1}(\mu \rho) J_{0}(\mu r)\right) \tau_{(\alpha+2) / 2}(r) d r
$$

Используя для оценки правой части этого равенства рассуждения, аналогичные проведенньп при $\nu=0$, получим $W_{1}(\rho, \mu)=O(1) \mu^{-\alpha} \rho^{-2}=O(1) \mu^{-\alpha} \tau_{1}(\rho)$.

Пусть $\nu=1 / 2$ и $1 \leqslant \alpha<2$. Из (12) и (13) имеем

$$
W_{1 / 2}(\rho, \mu)=(\rho \mu)^{-1} \int_{\rho}^{\infty} r \tau_{\alpha / 2}(r) \sin \mu(r-\rho) d r
$$

При $\alpha \in(1,2)$ вьполним в правой части последнего соотношения преобразование $\int_{\rho}^{\infty}=\int_{0}^{\infty}-\int_{0}^{\rho}$ и учтем, что в силу формул $(1.12 .41)$ и $(2.12 .49)$ из $[9$, c. 53,101$]$ и $(22)$ из $[10$, с. 76$]$

$$
\begin{aligned}
\int_{0}^{\infty} r^{(\alpha-1) / 2} K_{(3-\alpha) / 2} \cos \mu r d r= & 2^{(\alpha-3) / 2} \Gamma\left(\frac{\alpha-1}{2}\right)\left(1+\mu^{2}\right)^{(1-\alpha) / 2} \\
& \times F\left(-\frac{1}{2}, \frac{\alpha-1}{2} ; \frac{1}{2} ; \frac{\mu^{2}}{1+\mu^{2}}\right) \\
\int_{0}^{\infty} r^{(\alpha-1) / 2} K_{(3-\alpha) / 2} \sin \mu r d r= & \sqrt{\pi} 2^{(\alpha-3) / 2} \Gamma\left(\frac{\alpha}{2}\right) \mu\left(1+\mu^{2}\right)^{-\alpha / 2}
\end{aligned}
$$

Из асимптотических оценок окончательно получим, что $W_{1 / 2}(\rho, \mu)=O(1) \mu^{-\alpha} \rho^{-1}$, если $1<\alpha<2$. 
При $\alpha=1$ перепишем (34) с учетом (6) в виде

$$
\begin{aligned}
W_{1 / 2}(\rho, \mu)= & \int_{\rho}^{\infty} r^{-1} \sin \mu(\rho-r) d r+\int_{0}^{\rho}\left(K_{1}(r)-r^{-1}\right) \sin \mu(r-\rho) d r \\
& +\cos \mu \rho \int_{0}^{\infty}\left(K_{1}(r)-r^{-1}\right) \sin \mu r d r-\sin \mu \rho \int_{0}^{\infty}\left(K_{1}(r)-r^{-1}\right) \cos \mu r d r \\
\equiv & I_{1}+I_{2}+I_{3} \cos \mu \rho-I_{4} \sin \mu \rho .
\end{aligned}
$$

Так как [8, с. 149] $I_{1}=\sin \mu \rho \operatorname{Ci}(\mu \rho)-\cos \mu \rho \operatorname{si}(\mu \rho)$, в силу оценок для интегральных синуса и косинуса $\left[8\right.$, с. 150] имеем (здесь и далее $\gamma_{0}-$ постоянная Эйлера) $\left|I_{1}\right| \leqslant$ $|\sin \mu \rho|\left(\gamma_{0}+\ln \mu \rho+O(1)\right)+|\cos \mu \rho|(\pi / 2+O(1))=O(1)$.

Из асимптотики $K_{1}(z)$ при $z \geqslant 1\left[8\right.$, с. 17] вытекает, что $K_{1}(z)-z^{-1}=O(z \ln (e / z))$, $z \leqslant 1$. Поэтому при $\rho \leqslant 1 I_{2}=O(1)$.

Далее в силу (2.2.3) и (1.12.48) из [9, с. 65, 101] $I_{3}=(\pi / 2)\left(\mu\left(1+\mu^{2}\right)^{-1 / 2}-1\right)=O(1)$.

Наконец, $I_{4}=\lim _{\varepsilon \rightarrow 0+0} I(\varepsilon)$, где

$$
I(\varepsilon)=\int_{0}^{\infty}\left(K_{1-\varepsilon}(r)-r^{\varepsilon-1}\right) \cos \mu r d r
$$

Последний интеграл в силу формул $(1.3 .1)$ и (1.12.39) из [9, с. 19, 52] равен

$$
\begin{aligned}
\pi((\mu+ & \left.\left(1+\mu^{2}\right)^{1 / 2}\right)^{1-\varepsilon}+\left(\mu+\left(1+\mu^{2}\right)^{1 / 2}\right)^{\varepsilon-1} \\
& \left.-(\Gamma(1-\varepsilon))^{-1} 2 \mu^{-\varepsilon}\left(1+\mu^{2}\right)^{1 / 2}\right)\left(4 \sin \frac{\pi \varepsilon}{2}\left(1+\mu^{2}\right)^{1 / 2}\right)^{-1}
\end{aligned}
$$

Применяя правило Лопиталя, придем к равенству

$$
I_{4}=\gamma_{0}+\ln \frac{\mu}{\mu+\left(1+\mu^{2}\right)^{1 / 2}}+\left(\left(1+\mu^{2}\right)^{1 / 2}\left(\mu+\left(1+\mu^{2}\right)^{1 / 2}\right)\right)^{-1} \ln \left(\mu+\left(1+\mu^{2}\right)^{1 / 2}\right)
$$

откуда $I_{4}=O(1)$ при $\mu \geqslant 1$.

Автор выражает благодарность В.А. Ильину и В. С. Серову за внимание к результатам настоящей работы. 


\section{СПИСОК ЦИТИРОВАННОЙ ЛИТЕРАТУРЫ}

[1] Цикон Х., Ф резе Р., Кирш В., Саймон Б. Операторы Шрёдингера с приложениями к квантовой механике и глобальной геометрии. М., 1990.

[2] Simon B. Schrödinger semigroups // Bull. Amer. Math. Soc. 1982. V. 7. № 3. P. 447-526.

[3] Крицков Л.В.Аналитическое описание упорядоченного спектрального представления пространства $L_{2}\left(\mathbb{R}^{N}\right)$ относительно оператора Шрёдингера с потенциалом класса Като // Диффференц. уравнения. 1995. Т. 31. №12. С. 2038-2045.

[4] Ильин В. А., Антониу И. Равномерная на всей прямой оценка отклонения от разлагаемой функции ее спектрального разложения, отвечающего оператору Шрёдингера с ограниченным и измеримым потенциалом // Дифференц. уравнения. 1995. Т. 31. №10. С. 1649-1657.

[5] Ильин В. А., Крицков Л.В. Равномерная на всей прямой $\mathbb{R}$ оценка скорости сходимости спектрального разложения, отвечающего оператору Шрёдингера с суммируемым потенциалом // Диффференц. уравнения. 1996. Т. 32. № 1. С. 32-36.

[6] Денисов С. А. Равномерная на всей прямой $\mathbb{R}$ оценка скорости сходимости спектрального разложения, отвечающего оператору Шрёдингера с потенциалом из класса Като // Дифференц. уравнения. 1997. Т. 33. № 6. С. 754-761.

[7] Крицков Л.В. Оценка снизу образов Фурье по системе фундаментальных функций одномерного оператора Шрёдингера с суммируемым потенциалом // Дифференц. уравнения. 1997. T. 33. № 10. С. 1321-1328.

[8] Бейтмен Г., Эрдейи А. Высшие трансцендентные функции. Т. 2. М., 1966.

[9] Бейтмен Г., Эрдейи А. Таблицы интегральных преобразований. Т. 1. М., 1969.

[10] Бейтмен Г., Эрдейи А. Высшие трансцендентные функции. Т. 1. М., 1965.

[11] Бейтмен Г., Эрдейи А. Таблицы интегральных преобразований. Т. 2. М., 1970.

Московский государственный университет им. М.В.Ломоносова 